\title{
On the Images of Bodhi and Maitreya Statues in The Same Cave During the Tang Dynasty
}

\author{
Jiahua Jiang ${ }^{1, a}$ \\ ${ }^{1}$ Shenzhen Polytechnic. Xili Town, NanShan District, Shenzhen, P.R.China. \\ ajjh@szpt.edu.cn
}

\begin{abstract}
In the Buddhist grottoes of Tang Dynasty, there is a rare combination of statues in Guangyuan and Bazhong's sculpture art, in Sichuan Province. The combination of statues is unique and special in that, there is totally nothing similar elsewhere in the country. This form of combination as in the topic is the so-called Bodhi and Maitreya statues in the same cave. Why is it in Guangyuan and Bazhong districts Sichuan Province that these statues were created? Why is it not in other areas? Look back upon the history, does this combination of statues have any other implications, religiously or politically, apart from the significance of the image art? That is what the author is trying to reveal and discuss in this paper.
\end{abstract}

Keywords: Tang Dynasty; Bodhi; Maitreya; Same Cave

盛唐时期菩提瑞像与弥勒像同窟式图像研究

\author{
蒋家华 $1, \mathrm{a}$ \\ ${ }^{1}$ 深圳职业技术学院. 西丽, 南山, 深圳, 中国. \\ ajjh@szpt. edu. cn,
}

中文摘要. 在盛唐时期的佛教石窟造像中, 有一种罕见的组合造像出现在四川广元、巴 中等地的造像艺术中。这种造像的组合之所 以罕见和特殊, 是因为除此以外在全国其它 地方绝无仅有, 这种组合形式即是题目中所 谓的菩提瑞像与弥勒像同窟或邻窟同时而 造。需要探明的是, 为什么在四川广元、巴 中等地出现了这些造像, 而没有在其它地域 出现。如果还原历史, 这种组合的造像除了 图像学的意义之外还有没有更深的其它意
味, 诸如宗教、政治等意图, 对于揭示这种 意义这正是笔者在本文中讨论的目的。

关键词：盛唐; 释迦瑞像; 弥勒像; 同窟

\section{1. 引言}

本文的讨论, 笔者主要从三个方面进行 展开。其一, 交待菩提瑞像的不同称谓, 并 从文献角度考察菩提瑞像的由来; 其二, 罗 列四例有关菩提瑞像与弥勒像并置图式组 合的遗存, 并从图像角度对这种造像进行简 
单的论述; 其三, 阐释此种图式组合造像的 宗教、政治符号学意义。需要指出的是, 关 于此组合造像图式的研究, 目前学术界关注 学者不多, 而仅作考古学论述的主要学者有 李玉珢、罗世平、雷玉华、王剑平等 [1]。 在这些学者的论文中, 他们的共同点几乎都 是借助这种造像组合来辨识菩提瑞像, 从而 考察菩提瑞像在四川广元、巴中等石窟造像 中存在的特殊事实。遗憾的是, 这些学者虽 然也注意到了这种造像组合图式的特殊性, 但是没有进一步讨论这种组合背后的宗教、 政治符号意味进行讨论, 本文的研究将弥补 这一图式组合中尚未引起学者关注的部分。

\section{2. 菩提瑞像的由来}

菩提瑞像, 又叫菩提树像、或菩提像, 顾名思义是指释迦于菩提树下证得无上正 等正觉的释迦造像。在菩提瑞像的塑造中, 往往称其趿跃台座为金刚座, 故又称金刚座 真容像 (加一真容的称谓, 表明佛像与释迦 的对等关系)。该像的形制大致为: 佛像结 趿跌坐 (方座或莲花座), 左手施禅定印放 于腹前, 右手施降魔 (触地) 印, 头戴宝冠, 手带宝钏, 胸配金饰不等, 呈菩萨装, 头顶 还塑以菩提树不等, 故该像又叫菩萨装降魔 成道像, 通常称为菩提瑞像, 该像传说为弥 勒菩萨亲面佛陀真容而施造 (见下页图 1)。

关于菩提瑞像的由来最权威的文献来 自《大唐西域记》卷 8 《摩揭陀国上》云: “精舍既成, 招募工人, 欲图如来初成佛像 （降魔成道，笔者注）……见精舍内佛像俨 然, 结加跌坐, 右足居上, 左手玫, 右手垂, 东面而坐, 肃然如在。座高四尺二寸, 广丈 二尺五寸, 像高丈一尺五寸, 两膝相去八尺 八寸, 两肩六尺二寸, 相好具足, 慈颜若真, 唯右乳上图莹未周。既不见人，方验神鉴， 众咸悲叹, 殷懃请知。有一沙门, 宿心淳质, 乃感梦见往婆罗门而告曰: “我是慈氏菩萨, 恐工人之思不测圣容, 故我躬来图写佛像。 垂右手者, 昔如来之将证佛果, 天魔来娆, 地神告至, 其一先出, 助佛降魔。如来告曰: “汝勿忧怖, 吾以忍力, 降彼必矣。”魔王 曰: ‘谁为明证?' 如来乃垂手指地, 言: “此有证。' 是时第二地神踊出作证, 故今 像手仿昔下垂。” 众知灵鉴, 莫不悲感。于
是乳上未周, 填则众宝, 珠媞宝冠, 奇珍交 饰。设赏迦王伐菩提树已, 欲毁此像, 既睹 慈颜, 心不安忍, 回驾将返, 命宰臣曰: “宜 除此佛像, 置大自在天形。”宰臣受旨, 惧 而叹曰: “毁佛像则历劫招殊, 违王命乃丧 身灭族, 进退若此, 何所宜行!”乃召信心 以为役使, 遂于像前横垒砖壁, 心惭冥闇, 又置明灯, 砖壁之前画自在天。功成报命, 王闻心惧, 举身生疮, 肌肤篗裂, 居未久之, 便丧没矣。”[2]这是玄寢（629-645 西行 期间）在游历摩揭陀国佛教圣地菩提伽耶, 朝圣菩提瑞像时听闻到的关于该瑞像的传 说。从引用文献的内容看, 主要描述了菩提 瑞像的由来、形制, 及其神显等。首先, 文 献中叙述了该瑞像的由来, 是由婆罗门生感 梦到弥勒菩萨造菩提树像的神迹。其次, 介 绍菩提瑞像的形制为: 结跏跌坐, 右足居上, 左手玫, 右手垂, 东面而坐。座高四尺二寸, 广丈二尺五寸, 像高丈一尺五寸, 两膝相去 八尺八寸, 两肩六尺二寸。从瑞像的尺寸看, 应该是释迦等身像。另外, 由于弥勒在造 像时乳上未周, 则填配众宝、珠璎宝冠、奇 珍交饰, 正应了前面笔者所说的该瑞像的另 一个称谓“菩萨装降魔成道像”的装饰特征。 这则神话故事还有一关键之处, 即作为释迦 后补佛的弥勒佛, 成为了造作菩提造像的匠 作。

\section{3. 菩提瑞像与弥勒瑞像同窟、邻窟并置图 式}

到目前为止, 菩提瑞像与弥勒像同窟或 邻窟造作并置的遗存, 包括文献记载在内, 一共有四例。其中两; 例是同窟, 另两件是 邻窟。而在这四例中, 有一件是存文献记载, 现已不存。另三件遗存则存在与当下的石窟 造像遗存中。下面分别作出介绍, 先从邻窟 叙述开始。

第一例古遗存资料来自张彦远《历代名 画记》卷 3: “敬爱寺……佛殿内菩提树下 弥勒菩萨塑像, 麟德二年 (665) 自内出, 王玄策取到西域所图菩萨像为样, 东间弥勒 像, 西间弥勒像…… ” [3] 菩提瑞像的相 邻房间塑弥勒像, 如果单纯的从宗教意义来 看, 把弥勒菩萨 (或弥勒佛) 作为释迦之后 的继承者未来佛来看的话, 这种组合甚为合 
理。

第二例为广元菩提瑞像窟 (第366号窟) [4]（见下图2），雕業于712年。该窟为一 佛二弟子二菩萨造像二力士像, 主尊菩提瑞 像前有二趾姿供养菩萨, 环壁雕十二弟子。 其左侧 365 号窟为中心坛式窟, 坛上造一佛 二弟子二菩萨二力士, 主尊为弥勒 (见下图 3) 。据考证, 第365号窟、第366号窟为同 时开業的组合窟, 也就是笔者所谓的菩提瑞 像与弥勒造像相邻并置窟, 其目的也是要体 现释迦与弥勒的特殊关系。
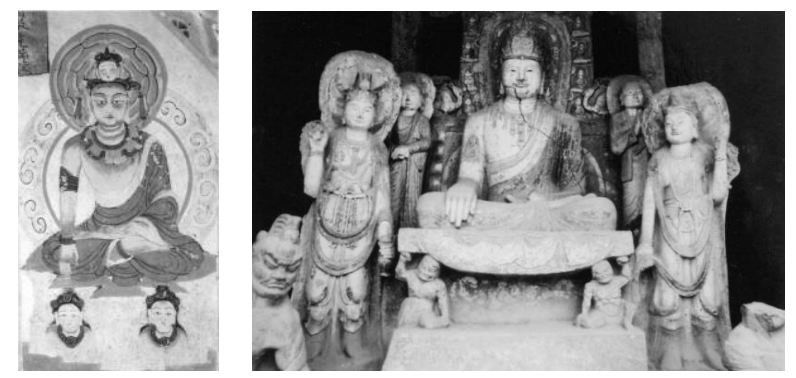

图 1 中唐莫高窟 237 西壁佛毞东坡菩提瑞像画(左) 图 2 广元千佛崖第 366 号窟菩提瑞像主尊 (右)

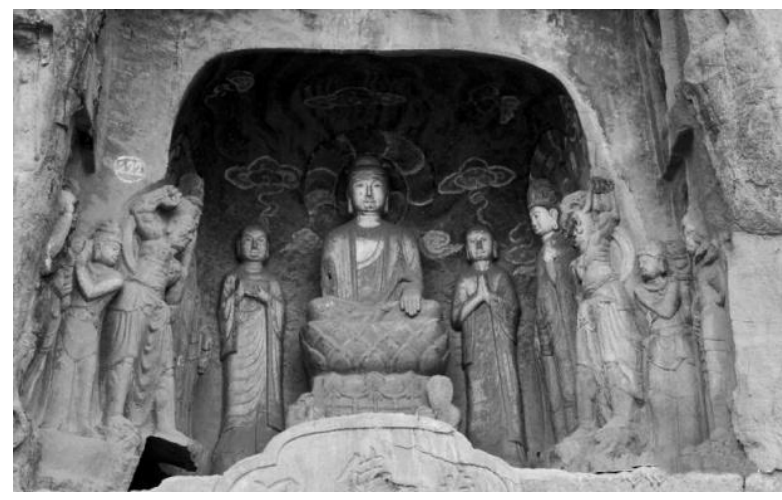

图 3: 广元千佛崖第 365 号窟弥勒主尊（右一）

第三件和第四件遗存是菩提瑞像与弥 勒造像同窟的例子, 分别为盛唐巴中西龛第 73 号窟 (左侧主尊为菩提瑞像式造像, 右 侧主尊为弥勒像, 见图 4)、盛唐巴中石门 第 12 号鿖 (右侧主尊为菩提瑞像式造像, 左侧主尊为弥勒像, 见图 5)。两窟的辅助 造像配置均为二佛、二菩萨、二弟子。

通过上述四则关于菩提瑞像式造像与 弥勒相邻或同窟而造的案例, 除《历代名画 记》所载的东京敬爱寺中菩提瑞像案例外, 其他三则均处于四川广元、巴中地区。因此, 在后面的论述中, 笔者将就这样的二佛并置 式石窟造像图式做出符号学意义上的阐释。 这种阐释将解释这种组合背后的多种事项
的隐秘含义。

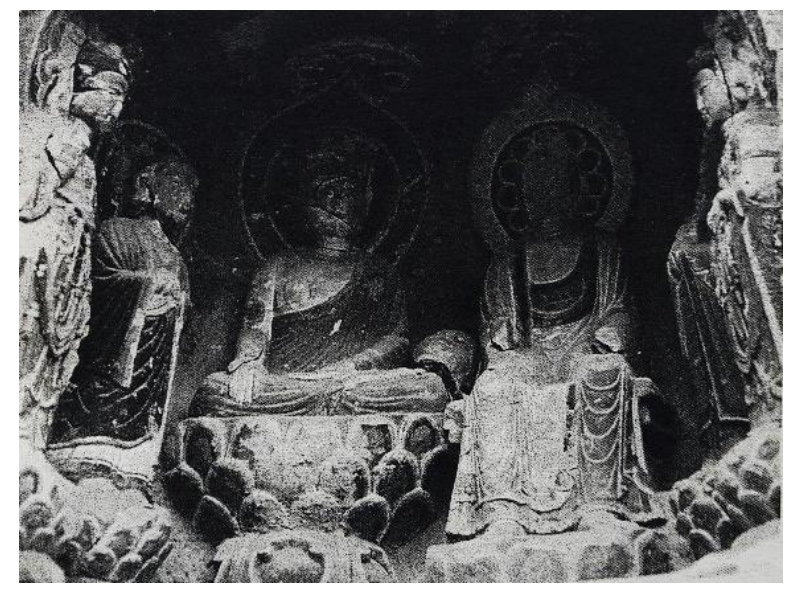

图4 盛唐四川巴中西龛释迦弥勒同龛 第73窟

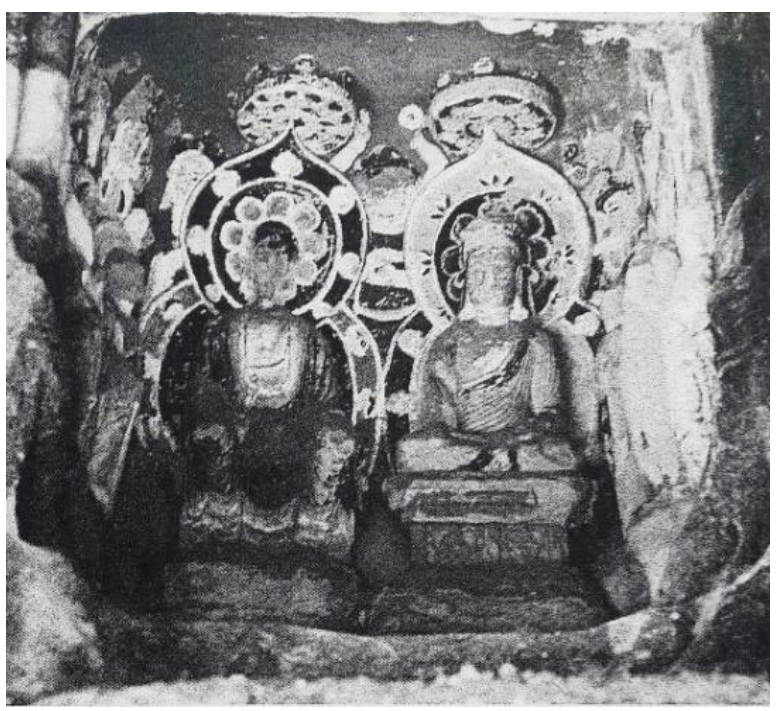

图5＼cjkstart盛唐四川巴中石门释迦弥勒同拿 第12窟

\section{4. 菩提瑞像与弥勒造像并置式图像的符号 象征}

根据上面的讨论我们知道, 菩提瑞像主 要指的是释迦结跏跌坐在菩提树下证得菩 提、觉悟, 战胜魔王波旬 (实际上是指心魔, 笔者注) 的瞬间画面, 呈现出一种降魔成道 式的图像: 即左手施禅定印置于腹前, 右手 施指地降魔印。这里提到菩提瑞像, 其本质 上是释迦的替身, 具有释迦替身的意味。[5] 对于同窟的弥勒造像而言, 作为像主的弥勒 在玄焋著《大唐西域记》卷 8 《摩揭陀国》 中, 被描述为是菩提瑞像的造作者、塑造者。 另外, 在佛教常识中, 弥勒菩萨作为释迦授 记的未来佛, 在龙华三会时下降到娑婆世界 替代释迦说法。因此, 此处的二佛并置则具 有重要的符号学隐喻性质。 
首先, 在笔者看来, 这种组合造像具有 隐喻武周朝及其前后时期政治格局的意味。 具体表现为, 菩提瑞像与弥勒造像的并置图 式暗示的是武周朝的高宗李治与皇后武则 天, 作为 “二圣” 的存在。菩提瑞像指涉的 是高宗, 弥勒指涉的是武后。关于武后化身 弥勒的传闻在《旧唐书》列传第四十三《张 仁愿传》云: “时有御史郭霸上表称则天是 弥勒佛身, 凤阁舍人张嘉福与洛州人王庆之 等请立武承嗣为皇太子, 皆请仁愿连名署 表, 仁愿正色拒之, 甚为有识所重。” [6] 另外, 又在《旧唐书》列传第一百三十三《外 戚》重还说: “怀义与法明等造《大云经》, 陈符命, 言则天是弥勒下生, 作阎浮提主, 唐氏合微。故则天革命称周, 怀义与法明等 九人并封县公, 赐物有差, 皆赐紫袈娑、银 龟袋。其伪《大云经》颁于天下寺, 各藏一 本, 令升高座讲说。”[7]应该说这两件事 是发生在高宗死后 (683) 发生的, 武则天 改国号为周（690）之间的事情。武则天利 用自己是弥勒菩萨转生的社会舆论, 进行革 命这是不争的史实。因此当时的个别佛教势 力为迎合武则天便大肆散播此等與论, 使得 坊间 “信以为真” , 这是常识。在武则天登 上帝位之后, 她 “自认为” 受命于佛 (或菩 提瑞像), 为弥勒下生, 并于长寿二年 (693) 九月加尊号 “金轮圣神皇帝” , 天册万岁元 年 ( 695) 加尊号为 “慈氏越古金轮圣神皇 帝”, 明确宣布自己就是转轮圣王、弥勒菩 萨的化身。 [8]

众所周知, 在武则天革唐为周的过程 中, 她正是利用了弥勒信仰的契机成功实现 了她的政治野心。有了以上的政治背景因 素, 再看上面笔者例举的四例菩提瑞像与弥 勒造像并置图像的政治象征意义, 则更加明 显。因此, 可以认为这些并置式造像实际上 是民间对这种政治格局的回应。

其次, 在这四个案例中, 其中三件遗存 出现在四川广元、巴中地区。笔者认为, 这 一地区出现菩提瑞像与弥勒并置的信仰, 与 该地区民众崇拜武后相关。因为信众认为, 作为武后的出生地利州 (现四川广元, 巴中 亦在广元附近), 理当进行造像崇拜。最为 重要的是, 于四川广元摩崖石刻第366窟中, 在菩提瑞像窟右壁处有造像碑《大唐利州刺
史毕公柏堂寺菩提瑞像颂》，碑文曰：“大 唐利州刺史毕公柏堂寺菩提瑞像颂并序/侄 前乡贡进士彦……化由乎觉忍/诚信资乎胜 根理实然也利州柏堂寺往居列城州牧做宅 天后圣帝……因寺以兴号/假树以立名初者 天竺不众生思者象法能殚众巧所擬罕成上 届通士感念……泥不满备珍/饰而相好周圆 灵哉真颜今即遗制粤若季父银青光禄大夫 使持节利州…… ”

从碑文中可以发现一些重要信息。其 一, 菩提瑞像的雕業出于利州 (现广元市), 而利州是武则天出生的地方。[9]宋代王存 《元丰九域志》附录《新定九域志. 利州》 记载: “(利州) 皇泽寺, 有唐武后真容殿。 按武士 (武则天父亲, 笔者注) 为利州都督, 生皇后于此。”而《利州都督府皇泽寺唐则 天皇后武氏新庙记》石碑, 立于后蜀广政二 十二年 (959年)，文称：“寺内之庙，不知 所创之因，古老莫传，图经罕记。”[10] 其二, 碑文中出现了 “天后圣帝” 字样, 那 么这里的天后圣帝应该指的是武则天。但 是, 如果指的是武则天的话, 延和元年 (712) 时她已经死去多年。鉴于这个原因，相似的 一则斯坦因文书 “2424号” 《阿弥陀经》的 题记中也出现了相似的称谓 “天皇天后” , 题记中显示是在景龙三年 (709), 砺波护 认为题记中的“天皇天后”可能指的是中宗、 韦后。[11]因为在 “庚子, 上皇帝 (中宗) 尊号曰应天神龙，（韦）皇后尊号曰顺天翊 圣, 大赦天下, 改元为景龙 (实际是韦氏欲 效仿武则天而进加的封号, 笔者注)”。[12] 应该说, 砳波护的说法也有道理, 但是笔者 认为 《大唐利州刺史毕公柏堂寺菩提瑞像 颂》碑文中出现的 “天后圣帝” 指的应该是 武则天而非中宗与韦后。理由是, 唐朝利州 作为长安进入四川腹地的咽喉 (所谓金牛 道）, 且利州（广元）又是武则天的故乡, 在武则天登上皇位到其死后的较长时期, 在 利州兴起了对武则天崇拜的社会风气。利州 百姓虔诚地将武则天奉为神明, 顶礼膜拜, 明朝天启年间邑令陈鸿恩所作《皇泽寺书事 碑记》云: “蒙之灵宫, 管境所依, 祷祈必 验”, “其间以水旱灾之事, 为军民祈祷于 天后之庙者, 无不响应”。由此原因, 《大 唐利州刺史毕公柏堂寺菩提瑞像颂》文中 
“天后圣帝”应该指的就是武则天。更何况， 作为地理位置利州而言, 根本与中宗和韦后 没有半点关系。而作为碑文刊刻于菩提瑞像 窟壁, 自然具有特殊的寓意。

\section{5. 结束语}

对于菩提瑞像与弥勒造像并置式图像的 探讨, 笔者以为具有特殊的意义。这种探讨 的意义在于, 通过对艺术形态的研究, 进而 探究造像背后的宗教学意义以及相关的政 治背景。同时, 通过对历史在场感的研究, 我们发现这种造像图式实际上是与当时的 政治生态相关。从系统化的观点看, 任何物 象的历史, 只要放进当时的历史语境, 无论 是艺术的, 还是宗教的, 政治的, 以及民俗 的, 它们作为自身的小系统, 其实都是当时 整个文化系统的一部分, 这些小系统之间实 际上都有着自然的关联。因此, 就本文而言, 由艺术，宗教、历史、政治的多个角度对菩 提瑞像与弥勒造像并置式图像作综合讨论, 这是笔者的一种基本研究路径, 期待等多的 专家学者加入研究和讨论。

\section{致谢}

该文为国家社科基金重大项目“多卷本 《中国寺观文化史》”(项目编号: 13\&ZD079) 的阶段性成果。并对该项目团队对本文的建 议和支持表示诚挚的感谢。

\section{References}

[1] Li Yumin, Study on Xiangmo Decorative Buddha In the Tang Dynasty, the Journal of Imperial Palace Academic Quarterly, Vol.23, pp.39-90, 2006; Study on Bodhi Statues In Sichuan Province, Heritage Press, pp.548-561, 2007. Luo Shiping, Study on Guangyuan Qianfoya Bodhi figure, Art Research(1), pp.54, 1991. Lei
Yuhua \& Wang Jianping: Research of Bodhi Statues in Sichuan Province, Sichuan Cultural Relics(1), pp.85-91, 2004; Lei Yuhua \& Wang Jianping, Research of Bodhi Statues in Sichuan Province Again, The Imperial Palace Museum(6), pp.142-148, 2005.

[2] Xuanzhuang, Datang Westernregions, Vol.8, CBETA, T51, no. 2087, pp.916, a16-b16.

[3] Zhang Yanyuan, The Famous Paintings Through The Ages, Vol.3, Liaoning Education Press, pp.38, 2001 .

[4] Lei Yuhua \& Wang Jianping, Research of Bodhi Statues in Sichuan Province, Sichuan Cultural Relics(1), pp.90, 2004.

[5] Duan Yuming, Xiangguo Temple, Bashu Press, pp.96, 2004.

[6] Liu Xu, Old Tang Book, Vol.93, Zhonghua Press, pp.2981, 1975.

[7] Liu Xu: Old Tang Book, Vol.183, Zhonghua Press, pp.4742, 1975.

[8] Liu Xu, Old Tang Book, Vol.6, Zhonghua Press, pp.124, 1975.

[9] Han Sheng, Wu Zetian's family and the birth of new exploration, Journal of Xiamen University(Philosophy and Social Sciences)(1), pp.69-71, 2002.

[10]Zhang Mingshan \& Huang Zhanyue: Huang Zesi Temple survey in Guangyuan county Sichuan Province, Journal of Archaeology(7), 1960.

[11] Libo $\mathrm{Hu}$, Sui and Tang Dynasties Buddhist Culture, Shanghai Ancient Books Press, pp.50, 2004.

[12] Liu Xu, Old Tang Book, Vol.7, Zhonghua Press, pp.145, 1975. 